\title{
Méthode de modélisation des processus complexes réalisés par des auteurs autonomes. Application à l'orientation des personnes en situation de handicap
}

\author{
Modelling method of complex processes performed by autonomous \\ authors. Application to the orientation of people with disabilities
}

\author{
Anderson Fabian Mosquera Varela ${ }^{1,2}$, Lorraine Trilling ${ }^{3}$, Thibaud Monteiro ${ }^{4}$ \\ ${ }^{1}$ Ressourcial, France, anderson.mosquera@ressourcial.fr \\ ${ }^{2}$ Laboratoire DISP, Université de Lyon, INSA-Lyon, France, anderson.mosquera@insa-lyon.fr \\ ${ }^{3}$ Laboratoire DISP, Université de Lyon, INSA-Lyon, France, lorraine.trilling@insa-lyon.fr \\ ${ }^{4}$ Laboratoire DISP, Université de Lyon, INSA-Lyon, France, thibaud.monteiro@insa-Iyon.fr
}

RÉSUMÉ. Un modèle de processus doit garantir la représentation des différents acteurs qui participent au processus et la manière dont ils sont en relation les uns avec les autres. La modélisation de toutes les relations entre tous les acteurs devient complexe lorsque chaque acteur se comporte de manière autonome et son comportement doit être constamment adapté au comportement des autres acteurs afin de garantir l'objectif du processus global. Le processus d'orientation des personnes en situation de handicap (PSH) doit garantir aux personnes un accompagnement selon leurs besoins. Dans ce processus, nous pouvons trouver 3 grands acteurs autonomes, le PSH, la maison départementale des personnes handicapées (MDPH) et les établissements et les services médico-sociaux (ESMS). L'objectif de ce document est de proposer une méthode pour modéliser des processus avec des acteurs autonomes et présenter l'utilisation de cette méthode dans la modélisation du processus d'orientation.

ABSTRACT. A process model must ensure a representation of the different actors involved in the process and how they relate to each other. The modelling of all the relationships between all the actors becomes complex when each actor behaves independently (autonomous actors) and his behaviour must be constantly adapted to the behaviour of the other actors in order to guarantee the objective of the global process. The orientation process for people with disabilities (PSH) must guarantee people support according to their needs. In this process, we can find 3 main autonomous actors, the $\mathrm{PSH}$, the departmental office of people with disabilities (MDPH) and social and medical-social institutions and services (ESMS). The purpose of this document is to propose a method for modelling processes with autonomous actors and to present the use of this method in the modelling of the orientation process.

MOTS-CLÉS. Modélisation de processus, BPMN, Processus complexes, Acteurs autonomes, Personnes en situation de handicap, Secteur médico-social.

KEYWORDS. Process modelling, BPMN, Complex processes, Autonomous actors, People with disabilities, Medico-social sector.

\section{Introduction}

En France, le secteur médico-social comprend une liste d'institutions et de services qui accompagnent les personnes en situation de vulnérabilité. Une partie de ces personnes vulnérables sont les personnes en situation de handicap qui nécessitent un accompagnement adapté à leur situation. Le processus d'orientation des personnes en situation de handicap cherche, à partir de l'analyse de la situation de la personne, à proposer une liste d'établissements qui peuvent accompagner la personne avec différents services et prestations.

Le processus d'orientation (figure 1) commence quand une Personne en Situation de Handicap (PSH) formule une demande à la Maison Départementale des Personnes Handicapées (MDPH). Cette demande contient plusieurs documents qui explicitent la situation de la personne et son besoin d'accompagnements. Un de ces documents est le «projet de vie » (PV), dans lequel la personne exprime ses souhaits et désirs. Une Équipe Pluridisciplinaire (EP), au sein de la MDPH, analyse la 
demande et propose, quand le cas le nécessite, une orientation vers une liste d'Établissements et de Services Médico-Sociaux (ESMS) adaptés aux besoins de la personne. Cette équipe peut traiter un peu plus de 200 dossiers d'orientation par mois, sans prendre en compte les autres demandes qui sont faites à la MDPH et qui nécessitent aussi l'intervention de l'EP [CNS 17]. À partir de cette analyse, la Commission des Droits et de l'Autonomie des Personnes Handicapées (CDAPH) envoie à la personne une notification précisant le refus ou l'acceptation du droit à l'accompagnement et en précise les raisons et modalités de soutien. La personne doit faire une demande d'admission à un ou plusieurs établissements explicités dans la notification de la CDAPH. Le processus d'orientation se termine lorsqu'un établissement démarre l'accompagnement de la personne ${ }^{1}$.

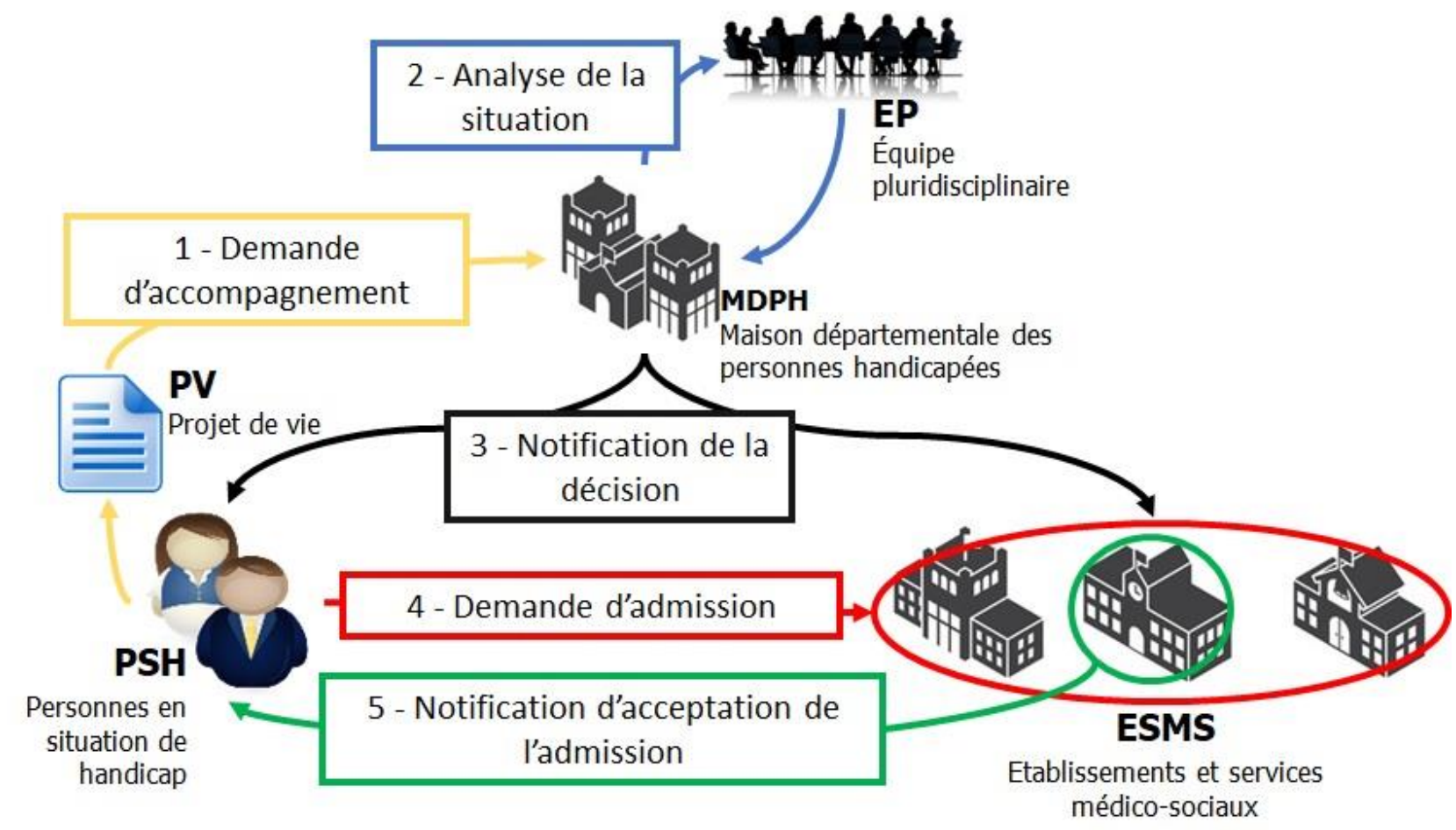

Figure 1. Processus d'orientation actuelle des PSH

Le processus actuel d'orientation contient certaines carences que nous citons ici :

- La demande formulée à la MDPH peut nécessiter plusieurs itérations.

- Selon la loi, la MDPH doit analyser la situation de la personne et donner une réponse en moins de quatre mois. Or, une étude montre que ces délais de traitement sont en moyenne, de 4 mois et 15 jours dans le cas des adultes et de 3 mois et 17 jours dans le cas des enfants [CNS 17]. La durée limite n'est donc pas toujours respectée.

- La liste des établissements proposés par l'EP à la personne n'est pas toujours adaptée pleinement aux besoins de la personne.

- Le temps d'attente entre la demande d'admission dans un établissement et son effectivité est important. Entre le moment où la personne reçoit la notification de la $\mathrm{CDAPH}$ et le moment où elle est admise dans un établissement, il peut se passer plus d'an.

-Une PSH peut avoir un accompagnement médico-social tout au long de sa vie. Parfois ces personnes restent de longues périodes sans accompagnement. Nous parlons alors de rupture du parcours d'accompagnement. Cette rupture a lieu lorsqu'une personne passe une période de temps sans aucun service ou prestation dont elle a besoin. Au cours de cette période, la situation de la personne

\footnotetext{
${ }^{1}$ En France, toute personne doit avoir une réponse d'accompagnement médico-social en lien avec ses besoins. Cette réponse est, en principe, indépendante de la situation financière de la personne et du mode de financement de celui-ci.
} 
peut se dégrader. Ce parcours se réfère aux services et aux prestations proposés à la PSH tout au long de sa vie, afin de lui offrir les outils nécessaires pour être autonome et avoir une vie la plus normale possible.

C'est notamment pour résoudre ces carences que le projet de recherche ACAPELHA (Accès et Continuité de l'Accompagnement des Personnes Enfants et aduLtes en situation de HAndicap) a été initié. Son objectif est d'améliorer le processus d'orientation en réduisant les difficultés mentionnées ci-dessus. L'objectif principal étant d'éviter la rupture du parcours d'accompagnement. Une des étapes du projet ACAPELHA est la modélisation du processus actuel d'orientation, du point de vue des acteurs de terrain. Cette modélisation cherche à : - caractériser le comportement détaillé de chaque acteur dans le processus; - identifier les difficultés de chaque acteur et leurs causes ; - lister les améliorations que chaque acteur considère comme nécessaires.

La modélisation du processus d'orientation implique d'être capable de gérer deux caractéristiques importantes du processus : l'autonomie de chaque acteur et la complexité des interactions.

La difficulté liée au niveau de l'autonomie décisionnelle de chaque acteur, fait référence à l'indépendance de chaque acteur sur son propre comportement au cours de l'exécution du processus. Dans le processus actuel, il n'y a pas d'entité dont le rôle serait de coordonner ces comportements individuels. Pourtant, l'Agence Régionale de Santé de chaque territoire (ARS) doit théoriquement garantir que le processus d'orientation respecte des standards nationaux, mais elle n'intervient pas dans la façon dont chaque acteur exerce ses activités.

La difficulté liée à la complexité du processus découle notamment de l'impact du comportement de chaque acteur sur le potentiel d'actions des autres acteurs du processus et sur son résultat final. Par exemple, lorsqu'un acteur n'envoie pas les informations nécessaires à un second acteur, il se peut que le processus ait un retard, ou que le second acteur décide de continuer sans ces informations, conduisant à un processus potentiellement dégradé. Cette dégradation peut impliquer un rejet inopportun ou une décision inadaptée à la demande par la MDPH.

Une remarque importante est que, du point de vue de l'application, la modélisation va permettre d'identifier les causes des différentes difficultés citées précédemment (figure 2). En effet, le modèle permettra de distinguer clairement les différentes relations entre les activités et les acteurs. Les caractéristiques du processus amènent à porter une attention particulière sur la méthode de modélisation utilisée.

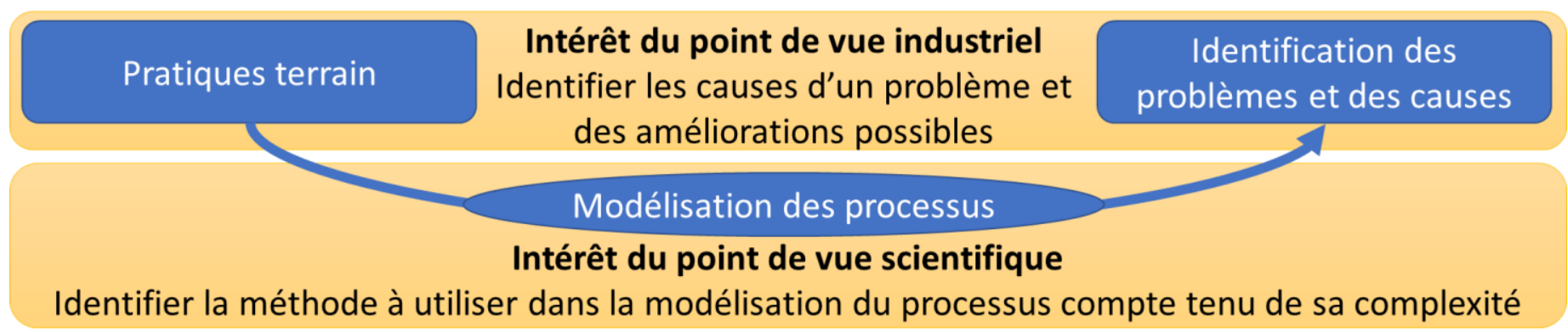

Figure 2. L'intérêt de modéliser le processus d'orientation

Dans ce document, nous allons proposer une méthode de modélisation d'un processus complexe au sein duquel agissent des acteurs autonomes. Nous commençons avec un état de l'art sur les différentes méthodes de modélisation et les caractéristiques des processus modélisés. Ensuite, chaque étape de la méthode proposée est expliquée et illustrée sur le cas de la modélisation du processus d'orientation. Pour finir, nous proposons une analyse et une conclusion sur cette méthode et sur sa mise œuvre dans le processus d'orientation. 


\section{2. État de l'art sur les méthodes de modélisation de processus}

Comme pour [DCU 05], l'objectif principal de la modélisation est la réingénierie des processus et des organisations. L'amélioration d'un système de production requiert au préalable de comprendre ses opérations et ses processus. Ainsi, la modélisation des processus est une étape fondamentale pour l'amélioration des performances. Il existe différents concepts de modélisation d'une organisation basés sur l'idée qu'il s'agit d'un outil puissant pour optimiser sa performance sous différents aspects [VER 99].

Dans le secteur de la santé, de nombreux projets ont été développés pour analyser et comprendre le fonctionnement d'un système afin de proposer une stratégie pour son amélioration [MAR 11]. La clé de la réussite de la modélisation est d'avoir une approche systémique, car elle prend en compte tous les aspects impliqués dans la prise de décision [VIL 14].

Les travaux autour de la meilleure façon de construire un diagramme de processus sont nombreux. [POL 17] présente une étude basée sur une revue systématique de la littérature, qui met en évidence les métriques de complexité à prendre en compte lors de la réalisation d'un diagramme de processus. [MEN 10] propose 7 lignes directrices à prendre en compte lors de l'élaboration d'un modèle de processus. On peut trouver plusieurs études sur les meilleures pratiques à prendre en compte dans la création d'un modèle de processus [CLA 17].

Un point à remarquer sur la littérature autour de la modélisation de processus est la facilité à trouver des documents relatifs aux bonnes pratiques à prendre en compte lors de la modélisation. Cependant, quand on parle de méthodes de modélisation pour des contextes spécifiques, comme c'est le cas d'un contexte complexe avec des acteurs autonomes, il y a peu de travaux qui considèrent les spécificités de ces contextes et la meilleure façon de les modéliser. Un exemple est celui de [OSO 14] qui propose une liste d'opérateurs à prendre en compte lors de la création d'un modèle de référence adapté à des organisations hétérogènes. Notre travail s'inscrit dans le champ des méthodes de modélisation dédiées à des contextes spécifiques. Notre contribution, du point de vue scientifique et méthodologique, est la proposition d'une méthode de modélisation apte à créer un modèle global d'un processus complexe, mettant en jeu des acteurs autonomes.

Lorsque nous parlons d'un modèle global avec des acteurs autonomes, nous faisons référence à un processus dans lequel chaque acteur est libre de définir comment il exécute ses activités. Cependant, son comportement a un impact sur le comportement des autres acteurs. Le modèle global devrait montrer le comportement de chaque acteur et comment celui-ci affecte les autres. Ce modèle global doit montrer comment le processus opérationnel de chaque acteur est impacté par les décisions opérationnelles prises par les autres acteurs et les informations transmises. Par exemple, la décision d'envoyer les informations données par la personne, au moment de sa demande, aux établissements ciblés. Ces informations peuvent éventuellement aider ces établissements à identifier lesquels sont les plus adaptés à la demande.

Le problème de la création d'un modèle qui représente le comportement de chaque acteur et sa relation avec les autres peut être associé au problème de modélisation de processus collaboratif [MU 15]. Les processus collaboratifs font référence à plusieurs entités qui interagissent dans un but commun mais avec un niveau d'autonomie au moment de la réalisation de leurs tâches. Dans notre cas, le but commun des acteurs (PSH, MDPH et ESMS) est de trouver une proposition de solution rapide et précise aux besoins de la personne qui nécessite un accompagnement. 


\section{Le langage de modélisation utilisé dans la modélisation du processus d'orientation de personnes en situation de handicap}

La méthode proposée dans ce document cherche à caractériser les différentes étapes à suivre pour modéliser un processus complexe avec des acteurs autonomes. Il est à noter que la structuration de la démarche n'est pas directement liée au langage de modélisation utilisé. La modélisation d'un système est une représentation de sa structure et de son fonctionnement. Elle permet la compréhension du fonctionnement du système pour servir de cadre à toutes les actions d'amélioration envisagées [TRI 06]. Trilling explique que le mode de modélisation doit être tiré par les objectifs attendus du modèle : meilleure compréhension du système, meilleure communication entre acteurs, évaluation des performances, analyse des dysfonctionnements, simulation du comportement du système (pour le dimensionnement des ressources, par exemple). Dans le cas étudié, nous cherchons à créer un modèle du processus d'orientation, qui permettra: (1) d'expliquer les interactions entre tous les acteurs et d'identifier le flux d'informations dans l'ensemble du processus et les moyens utilisés ; (2) d'identifier les points critiques du processus qui peuvent provoquer une rupture du parcours d'accompagnement ou une proposition d'orientation inadaptée; (3) de trouver les points d'améliorations qui pourraient améliorer la qualité de la proposition faite à la personne en demande.

Un autre aspect à considérer dans la modélisation, sont les points de vue que nous cherchons modéliser. Nous nous positionnons dans la perspective des travaux de Vernadat [VER 99]. Il existe quatre points de vue de modélisation principaux : le point de vue organisationnel, qui cherche à analyser le système de prise de décision d'une entreprise et la façon dont l'organisation est gérée ; le point de vue des ressources, qui cherche à répondre à la question «quelles sont les ressources nécessaires pour développer une activité ?»; le point de vue de l'information, qui a pour rôle principal la gestion des données utilisées dans l'organisation pour assurer la prestation du service ; et le point de vue fonctionnel d'une organisation, qui décrit ce que l'entreprise accomplit en termes de processus, d'activités et de tâches [ISO 06]. Dans notre cas, l'intérêt principal est le point de vue fonctionnel (la manière comment les activités du processus sont exécutées) et le point de vue informationnel (le flux d'informations tout au long du processus).

Une fois précisés les objectifs de la modélisation, il faut déterminer le langage de modélisation à utiliser. Au fil des ans, de nombreux auteurs et organisations ont développé des langages, des méthodes, des outils et des cadres pour faciliter la modélisation des processus. Chacun de ces outils se spécialise dans la modélisation d'un ou plusieurs des différents aspects (organisationnel, fonctionnel, des ressources, de l'information) et considère les différents systèmes de l'organisation (système d'exploitation, système de décision, système d'information). Puisque nous ne pouvons pas intervenir dans le fonctionnement du processus, notre intérêt principal est la réalisation d'un modèle pour une meilleure compréhension du processus d'orientation et l'identification des améliorations. Le modèle doit être clair et facile à comprendre pour tous les acteurs concernés. Dans le Error! Reference source not found. sont cités certains travaux réalisés dans la modélisation d'entreprises selon [CHA 07].

En raison des caractéristiques du problème du processus d'orientation, on s'intéresse à la modélisation descriptive du processus d'orientation axée sur les aspects opérationnels de chaque étape $\mathrm{du}$ processus. À cette fin, les approches UML, BPMN ou IDEF0 sont des méthodes potentielles [CHA 07]. Compte tenu de la facilité de compréhension du langage BPMN (Business Process Modeling Notation) par les acteurs, celui-ci est le langage de modélisation que nous avons utilisé. En outre, BPMN est le résultat de plusieurs expériences avec nombreuses notations. Selon nous, ce langage consolide les meilleures idées de toutes ces notations pour créer une notation standard unique. Également, la notion de ligne de nage permet de relier une représentation des acteurs à la représentation du processus métier. Dans le cadre des objectifs de ce travail, c'est cette notion qui prime. Une autre raison d'utiliser BPMN est la possibilité de faire une connexion entre les modèles de processus métier développés et la représentation des exigences du processus par les systèmes conçus pour implémenter et exécuter ces mêmes processus [GEI 17]. 
Idef-0, SADT

Entité-Association, les approches objet avec OMT évolution vers UML et des flux de données (Data Flow Diagram).

PSL (Process Specification Language), TOVE (TOronto Virtual Enterprise)

CIMOSA, ARIS et des langages comme IDEF3, IEM, DEM, etc.

BPM (Business Process Modelling), BPML (Business Process Modelling Language) évolution vers BPDM (Business Process Definition Metamodel) et BPMN (Business Process Modelling Notation).
La modélisation fonctionnelle

La modélisation de systèmes d'information

La modélisation de processus axée sur la représentation de concepts complexes à partir de concepts primitifs (une approche ontologique)

La modélisation centrée sur les problèmes d'intégration en entreprises

La modélisation focalisée sur les processus opérationnels

\section{Tableau 1. Les langages pour la modélisation de processus selon Chapurlat}

\section{Méthode pour la modélisation d'un processus complexe avec des acteurs autonomes}

Après avoir fait une révision de la bibliographie sur la modélisation et en considérant les caractéristiques de notre problème, nous proposons une méthode de modélisation à mettre en œuvre lorsque le processus à modéliser est complexe et que les acteurs sont autonomes. La méthode proposée est structurée selon 4 étapes qui cherchent à recueillir toute l'information nécessaire pour la création d'un modèle qui regroupe tous les acteurs en montrant clairement les liens entre ceux-ci.

\section{1. Étape 1: Entretiens avec les différents rôles impliqués dans le processus de chaque acteur}

Il est important de noter qu'un acteur fait partie du processus à modéliser. Cet acteur est partie prenante d'un ensemble d'activités. Celles-ci sont réalisées à travers différents rôles. Généralement, un rôle réalise des activités en lien avec le niveau de compétences qui lui sont associées. Par exemple, un acteur dans le processus d'orientation peut être l'ESMS, qui intègre différents rôles faisant partie du processus comme : le directeur de l'établissement, le psychologue de l'établissement, le travailleur social de l'établissement, etc. Une fois identifiés les acteurs et les rôles, il faut établir un questionnaire pour chaque rôle. Pour la construction du questionnaire, nous suivons la démarche structurée d'IDEF0. Cette démarche établit que pour chaque activité faite par le rôle dans le processus, il est nécessaire de poser des questions autour des 5 axes présentés dans la Error! Reference source not found.

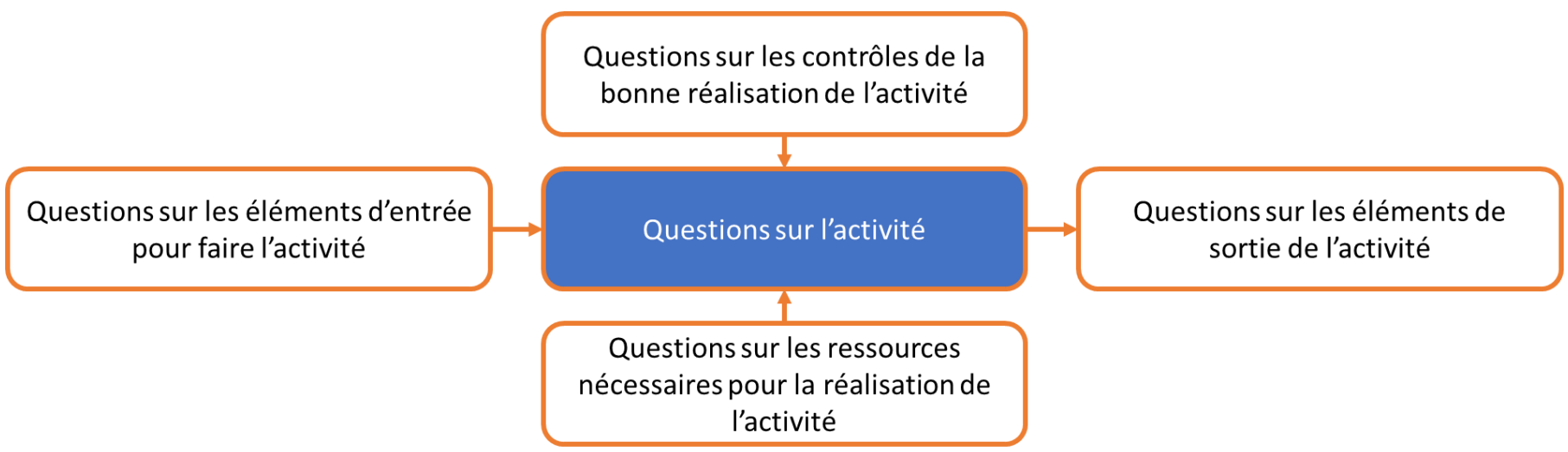

Figure 3. Guide pour la construction du questionnaire 
Dans la modélisation du processus d'orientation, l'étude de la réglementation et les échanges avec différents responsables d'ESMS nous permettent d'identifier trois acteurs clés du le processus d'orientation (Error! Reference source not found.).

- Tout d'abord, les personnes en situation de handicap et leurs proches : le processus d'orientation doit commencer et terminer avec eux, car le but de l'orientation est de proposer une solution personnalisée en fonction des leurs besoins. Il est nécessaire avant de commencer le processus d'orientation, d'identifier le besoin d'accompagnement.

- Un second acteur est la MDPH : cette agence a pour mission de recevoir, d'informer, d'accompagner et de conseiller les personnes handicapées et leurs proches, ainsi que de sensibiliser tous les citoyens au handicap. Cette organisation s'occupe de la partie la plus importante du processus d'orientation, qui consiste à proposer une liste d'ESMS adaptés à la personne en fonction de ses besoins.

-Enfin, un troisième acteur est les ESMS, regroupant les institutions et les travailleurs qui accompagnent les personnes handicapées. Comme précisé précédemment, chaque acteur a différents rôles qui participent au processus d'orientation des personnes en situation de handicap.

\begin{tabular}{|l|l|l|l|}
\hline Acteurs & $\begin{array}{c}\text { Personnes en situation } \\
\text { de handicap (PSH) et } \\
\text { leurs proches }\end{array}$ & \multicolumn{1}{|c|}{$\begin{array}{c}\text { Maisons départementales des personnes handicapées } \\
\text { (MDPH) } \\
\text { Personne en situation } \\
\text { handicap }\end{array}$} & $\begin{array}{c}\text { Directeur d'établissement } \\
\text { Services médico- } \\
\text { sociaux (ESMS) }\end{array}$ \\
\hline $\begin{array}{l}\text { Responsable légal de la } \\
\text { PSH }\end{array}$ & $\begin{array}{l}\text { Médecin (pédopsychiatre, médecin scolaire, médecin } \\
\text { MDPH) }\end{array}$ & $\begin{array}{l}\text { Directeur de } \\
\text { l'établissement }\end{array}$ & Éducateur \\
\hline \multirow{2}{*}{ Rôles } & Psychologue scolaire & Travailleur social \\
\hline & $\begin{array}{l}\text { Personnel dédié à l'emploi et à la formation } \\
\text { professionnelle (Cap Emploi, Psychologue du Travail } \\
\text { Pôle Emploi, référent « travailleurs handicapés » de Pôle } \\
\text { Emploi, etc.). }\end{array}$ & Psychologue \\
\hline & $\begin{array}{l}\text { Autres professionnels nécessaires pour analyser la } \\
\text { situation particulière et la personne et prendre une } \\
\text { décision. }\end{array}$ & \\
\hline
\end{tabular}

Tableau 2. Les rôles et les acteurs dans le processus d'orientation des personnes en situation de handicap

\section{2. Étape 2 : Création d'un modèle de processus de référence pour chaque acteur}

À partir de l'information récupérée des entretiens réalisés dans l'étape précédente, les modèles individuels du processus de chacun des acteurs peuvent être créés. Dans ce premier temps, ces modèles considèrent uniquement les activités faites par l'acteur concerné. Il est important de noter que, pour créer le modèle de processus d'un acteur, il faut réaliser auprès des différents rôles ${ }^{2}$ plusieurs entretiens. Pour garantir un meilleur recueil, ceux-ci sont à réaliser auprès de plusieurs acteurs du même type. C'est la raison pour laquelle nous utilisons ce que Osorio dans son article [OSO 14] nomme « opérateurs de transformation ». Ces opérateurs permettent de structurer la création d'un modèle de processus de référence pour des organisations hétérogènes (figure 4).

\footnotetext{
${ }^{2}$ L'utilisation du terme rôle renvoie aux activités réalisées par une personne impliquée dans une phase spécifique du processus. Par exemple, les activités d'évaluation faites par le psychologue au moment de l'admission dans un établissement sont un des rôles de l'acteur ESMS.
} 


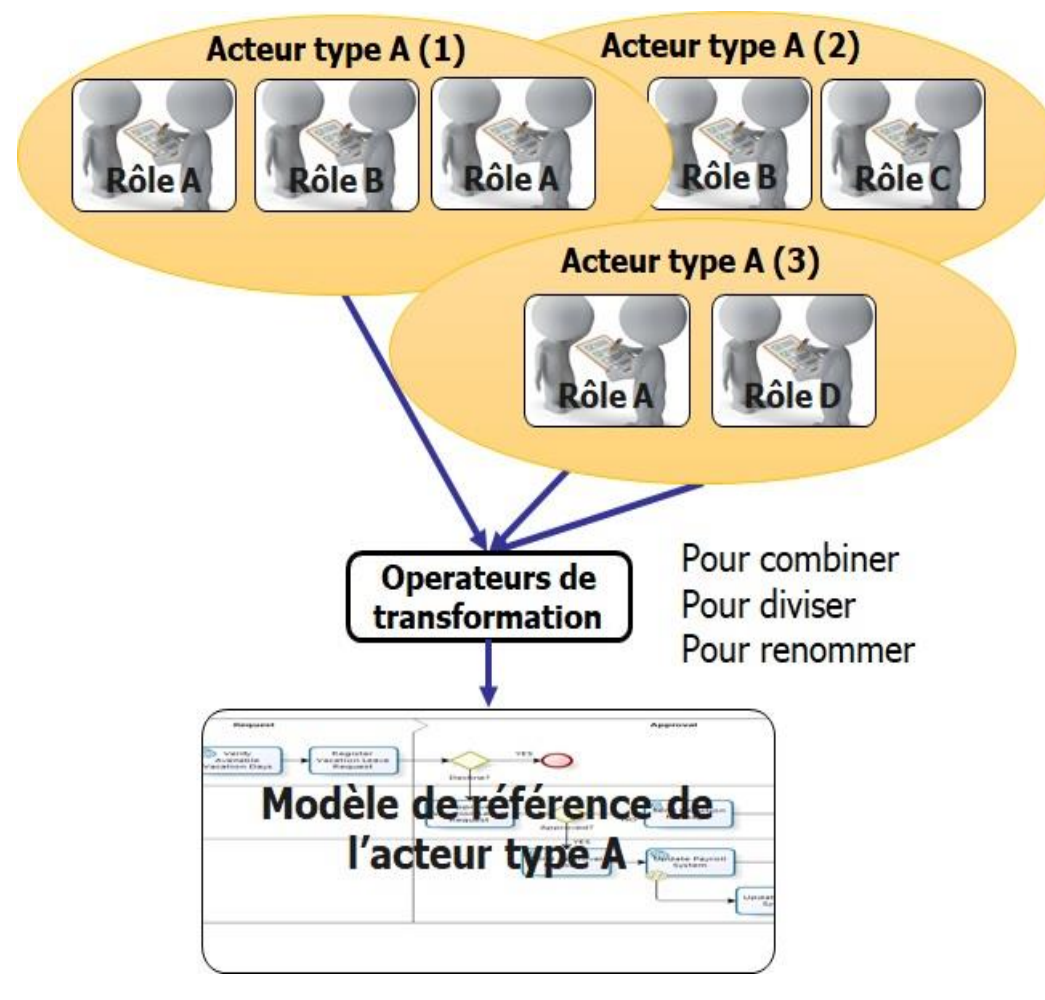

Figure 4. Représentation de la création du modèle de référence pour chaque acteur

Les opérateurs de transformation permettent à chaque acteur se reconnaître dans un cadre commun, aidant à identifier et définir les activités qui font effectivement partie du processus. Par exemple, la même activité peut être appelée différemment dans plusieurs organisations du même type ; dans ce cas, l'opérateur « renommer » permettra de définir un nom standard pour cette activité, en s'assurant que toutes les organisations identifient leurs activités dans le modèle. Dans le cas de l'opérateur « combiner», il y a des activités qui, au cours de l'étape d'entrevue, ont été identifiées comme différentes. Ces activités peuvent être combinées, au moment de la modélisation, pour en faire une seule activité, combinaison qui dépend de la complexité de celles-ci. Au contraire, dans le cas de l'opérateur " diviser », certaines activités, au cours des entretiens, ont été identifiées comme une seule activité. Mais étant donné la complexité de ces activités, il a été jugé plus pertinent de les diviser en plusieurs activités moins complexes. Cela permet une meilleure compréhension du modèle en maintenant le même niveau de granularité qui a été décidé.

Le modèle de référence de chaque acteur ne représente que l'ensemble des activités que cet acteur effectue dans le processus global de notre intérêt. Cela implique que lorsque les différents rôles sont interrogés, il est nécessaire de différencier clairement les activités réalisées par chaque rôle pour le processus étudié, et les activités que le rôle réalise, mais qui n'ont rien à voir avec le processus. Le caractère interactif, lors de la conception du modèle, permet que le modèle de référence de chaque acteur soit le résultat des convergences entre les différentes pratiques. Cette convergence est obtenue après avoir mené plusieurs entretiens avec différentes personnes qui participent au même processus et à réconcilier toutes ces informations dans le même modèle [OSO 14].

Un autre facteur important que nous devons considérer dans la création du modèle de référence est l'évaluation de toutes les activités effectuées par les différents rôles. Cela implique d'évaluer celles qui sont effectuées de manière ponctuelle pour faire face à des situations particulières. Il faut déterminer s'il est pertinent d'intégrer ces activités spécifiques au modèle de référence ou si ce comportement ne fournit pas d'informations pertinentes pour l'analyse. Par exemple, le cas d'une personne qui demande une orientation vers un établissement spécifique avec lequel elle est déjà entrée en contact.

Dans la modélisation du processus d'orientation, pour représenter le comportement de différents types d'acteurs, plusieurs entretiens ont été menés : (a) Modélisation du processus lié aux personnes 
handicapées et à leur famille immédiate : Entretiens avec 4 personnes en situation de handicap et 3 parents ; (b) Modélisation du processus lié à MDPH : Participation en tant qu'observateur à une commission d'orientation $\mathrm{CDAPH}$, entretiens avec un médecin coordinateur et un directeur d'établissement participant à l'EP de la MDPH; (c) Modélisation du processus lié aux ESMS: Entrevues avec 10 directeurs et gestionnaires, 1 psychologue, 1 travailleur social et 3 éducateurs spécialisés.

\section{3. Étape 3 : Validation du modèle de référence créé pour chaque acteur en considérant les informations obtenues dans la modélisation du processus des autres acteurs}

Lors de la phase de création du modèle de référence pour chaque acteur, il est possible de constater que les entrevues individuelles ne permettent pas de recueillir en une seule fois toutes les informations requises. Ce constat apparaît pendant la phase de modélisation, puisque c'est à ce moment qu'il est possible d'identifier l'information manquante et le type d'information concerné. Ainsi, l'étape de validation avec les acteurs permet de récupérer cette information. Elle permet aussi de vérifier que ce qui est modélisé est conforme aux pratiques métiers (figure 5).

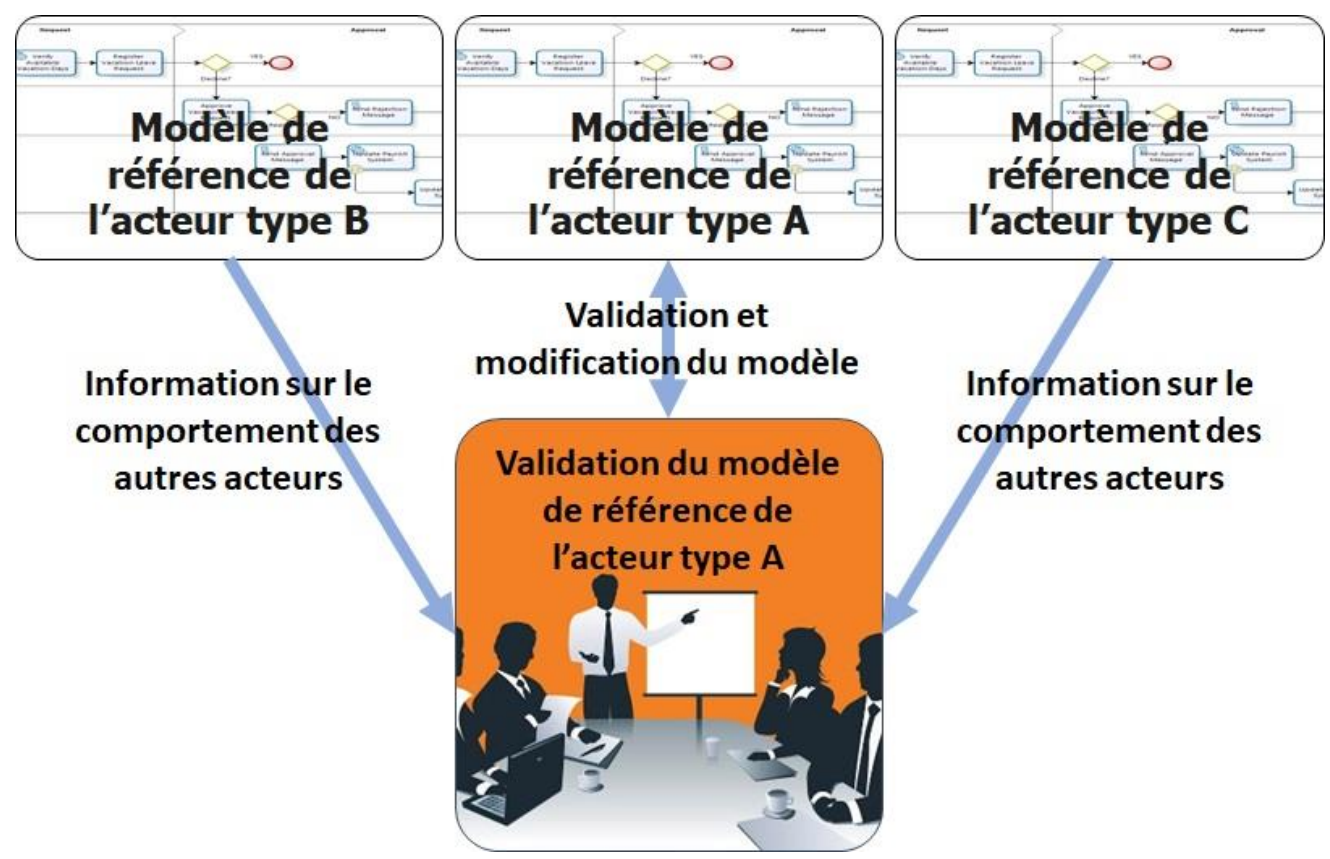

Figure 5. Représentation de la validation de chaque modèle de référence

Un autre élément important dans la validation du modèle de référence tient au fait qu'au fur et à mesure des entretiens, une connaissance sur les activités menées par les autres acteurs du processus est développée. Ainsi, il est possible de l'exploiter et de demander à chaque acteur quelle est sa réaction face aux différents comportements des autres acteurs du processus. Ceux-ci ont un impact direct sur ses propres activités. Un dernier élément exploitable dans la validation est la possibilité de modifier, durant la validation, le modèle de référence avec les différents rôles. Ceci est possible si le langage utilisé pour la modélisation est compréhensible par les personnes interviewées.

Dans la modélisation du processus d'orientation, la mise en place de cette étape dans la modélisation du processus d'orientation a nécessité, dans plusieurs cas, de valider le modèle avec des personnes différentes de l'étape 1 (les personnes interrogées), mais qui ont le même rôle dans le processus d'orientation. Cela a permis d'aborder les mêmes activités à partir de différents points de vue et d'identifier les bonnes et les mauvaises pratiques. Une bonne pratique peut être définie comme toute pratique qui peut accélérer le processus, en respectant tous les cadres juridiques et formels, avec un résultat final satisfaisant pour les acteurs impliqués dans le processus. 
4.4. Étape 4 : Créer un modèle global du processus qui permet de montrer tous les acteurs et les interactions

Après avoir modélisé le processus de chaque acteur et l'avoir validé avec les différents rôles de ces processus, la dernière étape consiste en la création d'un modèle global qui encapsule toutes les interrelations possibles (figure 6).

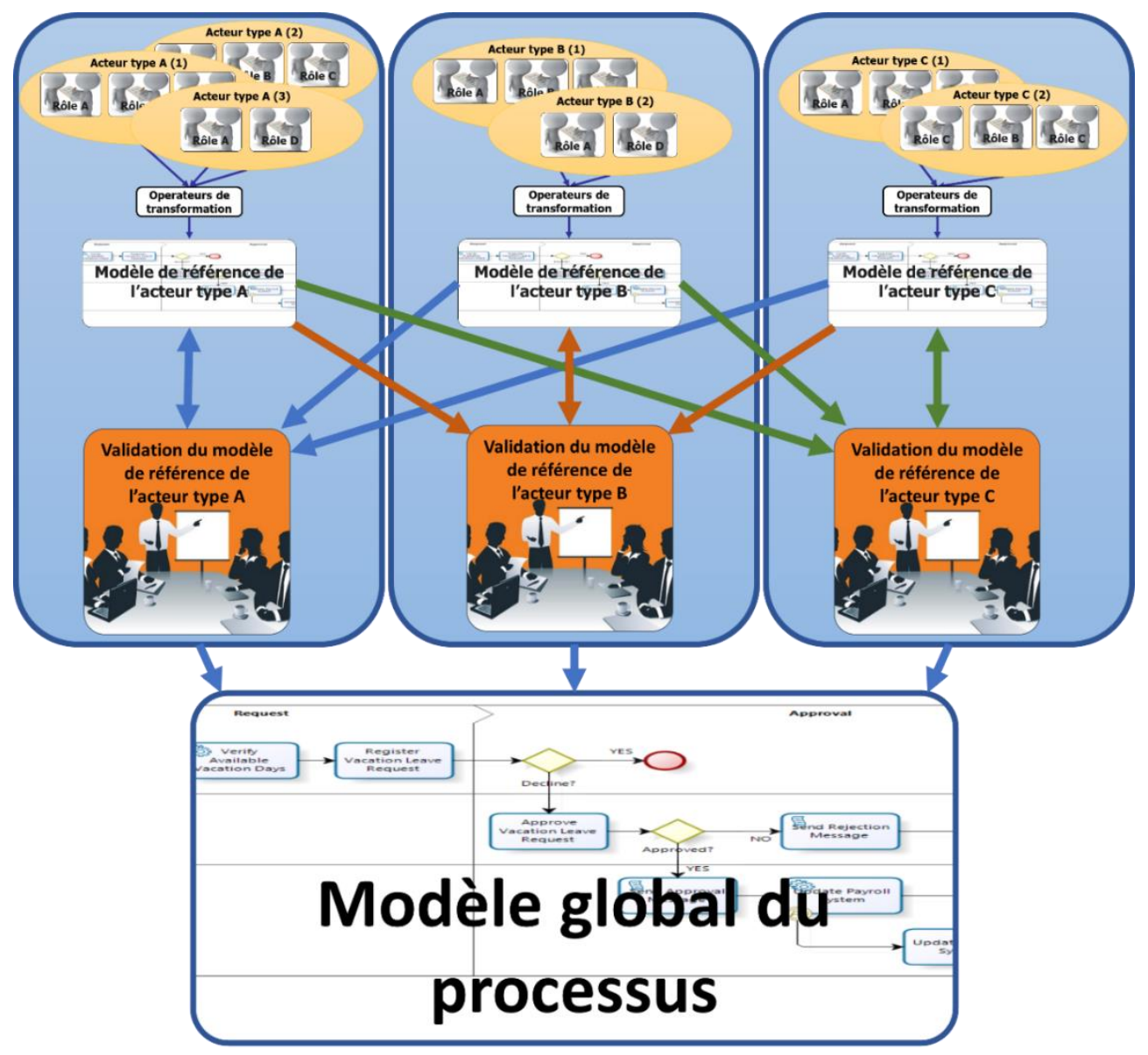

Figure 6. Représentation de la création d'un modèle global du processus

Il ne faut pas oublier que la modélisation est un outil de représentation du processus qui permet la prise de décision stratégique sur le processus. Donc, ce modèle global doit être en mesure de donner les informations nécessaires et suffisantes pour : comprendre le processus, identifier les différents points de difficulté et d'amélioration, identifier les différents échanges entre les acteurs.

Le modèle obtenu doit être équilibré dans le sens où il doit être suffisamment exhaustif pour représenter toutes les interactions entre les acteurs et les rôles, et suffisamment simple pour être compréhensible par tous.

Dans la modélisation du processus d'orientation, toutes les relations qui ont été préétablies, entre les acteurs dans les processus précédents, ont permis la création d'un modèle global du processus d'orientation. Vous pouvez consulter le modèle global du processus d'orientation sur le suivant lien : https://acapelha.ressourcial.fr.

La Figure 7 est le macro modèle du processus global d'orientation. Comme indiqué précédemment, le processus d'orientation a été modélisé en considérant le processus des 3 principaux acteurs et la manière dont ces acteurs sont liés. Ce modèle a été divisé en plusieurs étapes qui sont : l'expression de besoin, la constitution du dossier MDPH, la validation de celui-ci, l'analyse du dossier, la notification, la prise de contact et l'évaluation de l'admission. Un point important est que, à un certain moment du processus, les activités réalisées par la PSH sont menées conjointement avec les acteurs de l'ESMS. 


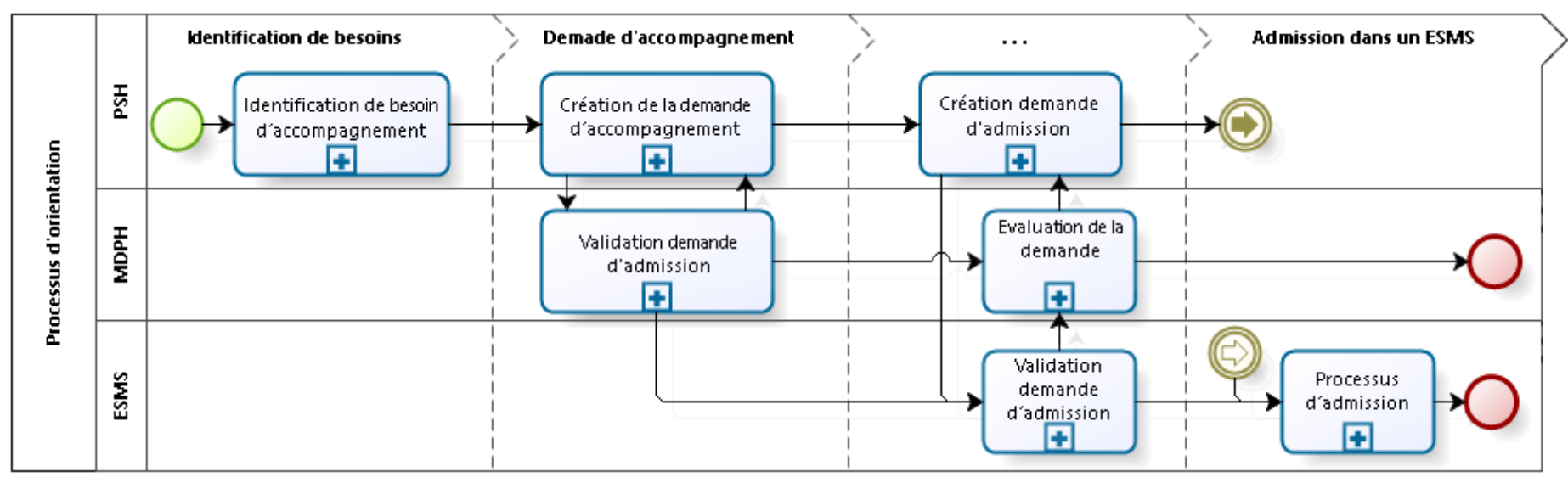

Figure 7. Macro modèle du processus global d'orientation

\section{Réflexion sur le processus d'orientation de personnes en situation de handicap}

La finalité de la modélisation d'un processus est de trouver, à partir de la connaissance acquise, les meilleures pratiques qui peuvent être mises en place. En considérant que le but du processus d'orientation est de proposer le plus rapidement possible à la personne, un accompagnement adapté à ses besoins, afin d'éviter que la personne ne soit laissée sans un accompagnement adapté (éviter la rupture du parcours d'accompagnement). Nous présentons une analyse des connaissances obtenues par la méthode proposée. Les principales causes des différents problèmes sont alors explicitées.

Quand le dossier de la personne est refusé dans sa première étape (avant l'analyse par l'EP), c'est généralement parce que les informations et/ou les documents qui étayent la demande ne sont pas suffisants. Une des causes principales est le manque de connaissances des éléments requis pour le dépôt d'un dossier à la MDPH. Le processus d'orientation doit commencer par l'identification des besoins d'orientation. Grâce au modèle réalisé, il a été possible d'identifier quels sont les différents acteurs susceptibles d'aider la famille. Pour les familles, aujourd'hui, il n'est pas simple d'identifier les acteurs qu'elles doivent, ou peuvent, contacter. Un exemple est le médecin traitant, qui peut diriger la personne vers les institutions appropriées lorsqu'il identifie un problème de handicap.

La raison principale pour laquelle la demande d'orientation n'est pas traitée en moins de 4 mois est le nombre important de demandes que l'EP doit étudier. De plus, cette situation de surcharge rend difficile la possibilité d'une personne à rencontrer à l'EP pour expliquer plus en détail sa situation (un droit normalement pris en compte dans la législation). Dans les faits, l'EP prend seule l'initiative et propose des entretiens seulement aux personnes lorsqu'il est nécessaire qu'elles apportent des informations complémentaires.

Un des problèmes du système actuel est le manque de compatibilité entre les besoins de la personne et les services proposés. Il existe plusieurs raisons :

- L'EP n'est pas à jour sur les informations relatives aux services proposés par les établissements ;

- La personne est dans une situation complexe, donc a besoin de services de différents types d'établissements (car les caractéristiques d'un seul établissement n'arrivent pas à répondre à la totalité des besoins) ;

- Le décalage entre le moment de l'expression du besoin et le moment de l'obtention de la réponse fait que cette réponse n'est plus forcément adaptée aux besoins actuels de la personne (la situation de la personne peut se dégrader avec le temps).

Une amélioration importante serait la possibilité d'augmenter le degré de finesse d'une proposition d'orientation en proposant des services adaptés aux personnes, et non pas des établissements. Cette 
amélioration implique que l'EP ait l'information sur les services et les établissements du territoire à jour.

Lorsque la CDAPH prend la décision de diriger la personne vers un établissement, elle envoie généralement une notification à la personne avec la liste des ESMS proposés par l'EP. Dans très rares cas, les ESMS sont informés de cette décision. Les ESMS prennent connaissance de la notification lorsque la personne les contacte. En considérant les nombreuses demandes d'admission que les établissements reçoivent, le partage des notifications entre ESMS est un point d'amélioration. Notifier simultanément les établissements et la personne permettrait aux établissements de prévoir le nombre de personnes susceptibles de formuler une demande d'admission.

Tous ces éléments sont susceptibles de laisser une personne sans accompagnement adapté. Et de la mettre dans une situation de rupture du parcours d'accompagnement. Il est donc important de trouver des solutions à ces problèmes.

Un autre problème du système actuel se situe au niveau du flux d'information entre les acteurs, comme :

- Une personne qui demande à la MDPH une orientation, doit considérer que du point de vue légal, le temps de réponse ne doit pas dépasser 4 mois (délai très peu respecté). Pendant cette période, la situation de la personne peut changer : i.e. admission dans un autre établissement, dégradation de la situation personnelle, etc. Le problème est que les personnes n'informent pas nécessairement ces changements à la MDPH.

- Lorsque la MDPH envoie à la personne une notification avec une liste de plusieurs ESMS, la personne peut commencer un processus d'admission dans un ou plusieurs ESMS, les ESMS doivent communiquer entre eux afin d'éviter de proposer la place dans un ESMS lorsque la personne a déjà trouvé une solution dans un autre ESMS.

- Parfois, certaines PSH font une première demande d'admission auprès d'un ESMS, mais y renoncent finalement. Dans ce cas, la MDPH devrait en être informée pour garder une trace de l'historique de la PSH et pourquoi cet ESMS ne lui convenait finalement pas.

Toutes ces difficultés mettent en évidence la nécessité de créer un système qui aide l'Équipe Pluridisciplinaire de la MDPH à prendre les décisions d'orientation. Ce système doit tenir compte des évolutions liées aux besoins de la PSH et aux offres de services des ESMS. En même temps, il doit permettre à tous les acteurs du processus de connaître l'état de chaque orientation.

\section{Conclusions sur la méthode et perspectives}

La contribution principale de ce travail est la proposition d'une méthode de modélisation apte à intégrer le comportement d'acteurs autonomes interagissant dans un processus complexe. Nous considérons les acteurs comme autonomes, car chacun prend ses propres décisions sur son comportement et qu'il n'y a pas de superviseur pour déterminer les actions de chacun. Nous considérons le processus comme complexe, car la façon dont chaque acteur exécute les activités du processus influence la manière dont les autres acteurs exécutent leurs activités. Il y a donc interdépendance dans les activités du processus.

L'étape la plus importante de la méthode de modélisation proposée vise à valider le modèle de référence de chaque acteur (étape 3). Pendant les entretiens avec les différents rôles, il est facile d'identifier les activités standards de chaque acteur dans un contexte idéal. En revanche, l'information qui n'est pas directement identifiable concerne les activités non standards. Elles sont relatives soit à des contextes spécifiques, soit à des comportements défavorables que cet acteur adopte. Ces informations sont toutefois identifiables en partant du point de vue des acteurs impactés par ces comportements. Ainsi, cette étape permet de questionner chaque acteur par rapport à ce que les autres considèrent 
comme des mauvaises pratiques. Elle permet aussi de questionner les acteurs sur la manière dont ces pratiques sont générées et si elles sont vraiment dérangeantes de leur point de vue.

La connaissance acquise par l'interviewer sur le processus permet aussi de faire ressortir lors de la phase de validation, et en interaction avec les acteurs, des éléments non explicites. Cela est particulièrement utile pour identifier les bonnes pratiques à faire émerger en pistes d'amélioration.

L'étape traitant de la création d'un modèle de processus global nécessite plus d'attention (étape 4). La représentation de toutes les activités des acteurs dans le même modèle de processus doit être suffisamment exhaustive pour modéliser toutes les interactions possibles entre les acteurs. En même temps, le modèle de processus global doit être suffisamment clair pour être compréhensible par tous les acteurs concernés.

Une prochaine étape à considérer serait la mise en œuvre de la méthode proposée dans d'autres contextes présentant les mêmes caractéristiques (acteurs autonomes participant à des processus complexes). Par exemple, le processus opérationnel de coordination et/ou de collaboration entre différents établissements qui proposent conjointement un accompagnement à une personne ayant un besoin complexe.

Une autre perspective autour de la méthode proposée est la possibilité d'une étape finale de validation du modèle global avec tous les acteurs en même temps. Ce serait un espace de discussion très intéressant pour identifier les améliorations dans une démarche plus globale de réingénierie des processus métiers.

\section{Remerciements}

Cette recherche a été financée par le gouvernement français à travers l'agence régionale de santé Auvergne-Rhône-Alpes (ARS AURA), dans le cadre du projet ACAPELHA.

\section{Bibliographie}

[CNS 17] CNSA, «Synthèse des rapports d'activité des MDPH 2016 ». Caisse nationale de solidarité pour l'autonomie, p. 47, 2017.

[DCU 05] Y. Ducq, J. C. Deschamps, et B. Vallespir, « Re-engineering d'un système hospitalier par l'utilisation de la méthodologie GRAI », J. Eur. des Systèmes Autom., vol. 39, no 5-6, p. 605-636, sept. 2005.

[VER 99] F. Vernadat, Techniques de modélisation en entreprise: applications aux processus opérationnels. Economica, 1999.

[MAR 11] M. Martin, R. Champion, L. Kinsman, et K. Masman, «Mapping patient flow in a regional Australian emergency department: a model driven approach », Int. Emerg. Nurs., vol. 19, nº 2, p. 75-85, avr. 2011.

[VIL 14] S. Villa, A. Prenestini, et I. Giusepi, «A framework to analyze hospital-wide patient flow logistics: evidence from an Italian comparative study », Health Policy, vol. 115, n 2-3, p. 196-205, avr. 2014.

[POL 17] G. Polančič et B. Cegnar, « Complexity metrics for process models - A systematic literature review », Comput. Stand. Interfaces, vol. 51, p. 104-117, mars 2017.

[MEN 10] J. Mendling, H. A. Reijers, et W. M. P. van der Aalst, « Seven process modeling guidelines (7PMG) », Inf. Softw. Technol., vol. 52, n 2, p. 127-136, févr. 2010.

[CLA 17] J. Claes, I. Vanderfeesten, F. Gailly, P. Grefen, et G. Poels, « The Structured Process Modeling Method (SPMM) what is the best way for me to construct a process model? », Decis. Support Syst., vol. 100, p. 57-76, août 2017.

[OSO 14] G. Osorio, L. Trilling, T. Monteiro, F. Albert, et P.-A. Millet, «A Method to Create a Process Reference Model Adapted to Heterogeneous Organizations », in IFIP WG 5.7 International Conference, 2014, p. 232-239.

[TRI 06] L. Trilling, « Aide à la décision pour le dimensionnement et le pilotage de ressources humaines mutualisées en milieu hospitalier », INSA Lyon - Institut National des Sciences Appliquées de Lyon, 2006. 
[ISO 06] ISO, «ISO 19439:2006 Enterprise integration -- Framework for enterprise modelling ». International Organization for Standardization, p. 41, 2006.

[CHA 07] V. Chapurlat, "Vérification et validation de modèles de systèmes complexes: application à la Modélisation d'Entreprise », Université Montpellier II - Sciences et Techniques du Languedoc, 2007.

[GEI 17] M. Geiger, S. Harrer, J. Lenhard, et G. Wirtz, « BPMN 2.0: The state of support and implementation », Futur. Gener. Comput. Syst., vol. 80, p. 250-262, mars 2018.

[MU 15] W. Mu, F. Bénaben, et H. Pingaud, «A methodology proposal for collaborative business process elaboration using a model-driven approach », Enterp. Inf. Syst., vol. 9, n 4, p. 349-383, mai 2015. 